\title{
Glaube und Wirtschaft Zur normativen Bedeutung des Ordo-Begriffes bei Walter Eucken
}

\author{
Giuseppe Franco
}

Feodor Lynen-Forschungsstipendiat bei der Alexander von Humboldt-Stiftung an der Universität Salento/Lecce und Privatdozent an der Theologischen Fakultät der Katholischen Universität Eichstätt-Ingolstadt

Received October 9 2016; Accepted September 112017

Zusammenfassung: Der Beitrag analysiert zunächst die Bedeutung des Ordo-Gedankens nach dem deutschen Nationalökonom Walter Eucken, dem Begründer des Ordoliberalismus der Freiburger Schule. Danach werden, im Anschluss an die Denkschrift des Freiburger Bonhoeffer-Kreises, die Rolle und die normative Dimension des christlichen Glaubens für das Denken Euckens sowie für seine ordnungsökonomischen und ordnungspolitischen Auffassungen untersucht. Dabei wird auch auf die Konvergenz zwischen Euckens Ordoliberalismus und der christlichen Sozialethik eingegangen.

Schlagwörter: Walter Eucken • Scholastik • Ordoliberalismus • Ordo-Gedanke • Katholische • Soziallehre • christliche Sozialethik • Wirtschaftsethik

(c) De Gruyter Open Sp. z 0.0 .

\section{Einleitung}

Ein zentraler mittelalterlicher Begriff, der erheblichen Einfluss auf den Ordoliberalismus der Freiburger Schule bis zur Sozialen Marktwirtschaft hatte, ${ }^{1}$ ist die Idee des Ordo-Gedankens. Die Geschichte dieses Konzeptes zeigt, dass dieser Gedanke verschiedene Ausprägungen und Dimensionen erlangt hat bzw. ihm unterschiedliche Bedeutungen zugeschrieben worden sind ${ }^{2}$ - in kosmologischer, metaphysischer, teleologischer, ontologischer, religiöser, ethischer, ökonomischer und politischer Hinsicht. Der Ordo-Gedanke bildet ein Verbindungsstück zwischen der Scholastik und dem Denken von Walter

1 In der wissenschaftlichen Diskussion wird weitgehend erkannt, dass das Programm des Ordoliberalismus der Freiburger Schule das theoretische und historische Fundament darstellt, aus dem später die Soziale Marktwirtschaft entstanden ist, ohne dabei die unterschiedlichen theoretischen Ansätze und die verschiedenen Akzentuierungen zu verkennen, die bei den Vertretern der beiden Konzeptionen auftauchen. Vgl. Goldschmidt und Wohlgemuth 2008; Starbatty 1997.

2 Vgl. Art. Ordnung, in: Ritter und Gründer 1984, S. 1249-1310.
Eucken (1891-1950), dem Begründer des Ordoliberalismus der Freiburger Schule. Gleichwohl unterscheiden sich die Ordo-Auffassungen. Es geht also um eine Idee, die innerhalb der wissenschaftlichen Literatur sowohl von Vertretern der christlichen Sozialethik, aber auch von Wirtschaftswissenschaftlern unterschiedlich interpretiert worden ist. Vor allem die an sich recht kurzen Ausführungen Euckens zum Ordo-Begriff haben eine Reihe von Kritiken und Stellungnahmen ausgelöst, die eine besondere Rekonstruktion und kritische Würdigung erfordern.

Zur Betrachtung der ökonomisch-soziale und die politische Situation der ersten Jahrzehnte des 20. Jahrhunderts, muss sich zunächst der historische Hintergrund angesehen werden. Die Verschärfung der internationalen Krise nach dem Ersten Weltkrieg, die Erfahrungen der Weltwirtschaftskrise von 1929, der Aufstieg der totalitären Regime wie Nationalsozialismus und Sowjetsozialismus, der Ausbruch des Zweiten Weltkriegs, die historischen Degenerationen des Kapitalismus und des Liberalismus sowie die 
Krise der wirtschaftlichen, gesellschaftlichen und politischen Lage und die erneut auftretende Soziale Frage zur Situation der Arbeiter zwingen Eucken - wie auch die anderen Vertreter des Ordoliberalismus - sich der Grundfrage zu stellen, wie eine menschenwürdige und gerechte Wirtschafts- und Gesellschaftsordnung aussehen sollte.

Euckens ordnungsökonomische Tradition und die christliche Sozialethik weisen deutliche Parallelen auf. Die inneren Bezüge beider Ansätze zeigen sich bereits terminologisch. Um sich vom wirtschaftlichen Liberalismus klassischer Prägung abzugrenzen, verwendet Eucken den Begriff des Ordo, der in der Folge geradezu konstitutiv für das wirtschaftspolitische Programm des Ordoliberalismus wurde. Auch das von Eucken und Franz Böhm im Jahr 1948 begründete „ORDO-Jahrbuch für die Ordnung von Wirtschaft und Gesellschaft" trägt diesen Begriff im Titel. Um die normativen Bezüge zwischen Ordoliberalismus und christlicher Sozialethik herauszuarbeiten, ist zunächst das Verständnis von Ordo in beiden Konzepten zu klären. Danach werden im Anschluss an die Denkschrift des Freiburger Bonhoeffer-Kreises die Rolle und die normative Dimension des christlichen Glaubens für das Denken Euckens und für seine ordnungsökonomischen und ordnungspolitischen Auffassungen untersucht.

\section{Vom Ordo-Gedanken des Mittelalters zum Ordo-Verständnis Walter Eucken}

Die Ordo-Idee bezeichnet im Allgemeinen ein Verhältnis einzelner Teilen zu einem Ganzen. In der scholastischen Theologie ist der Ordo-Gedanke vor allem mit der Schöpfungstheologie verbunden und dementsprechend mit der Auffassung, dass die ganze Wirklichkeit aus der Sicht Gottes zu verstehen ist und alle Teile der Wirklichkeit zu einer höheren und umfassenderen Ordnung, einem sinnvollen Ganzen gehören. Nach scholastischer Vorstellung ist die Welt kein Zufallsprodukt, sondern ein von Gott geschaffener, geordneter Kosmos. Die physische Welt besteht aus vielfältigen und unterschiedlichen Einzelgebilden, die jedoch in ein harmonisches, aufeinander bezogenes Gesamtgefüge gesetzt sind.

Der Ordo-Gedanke beschreibt die Annahme einer allumfassenden, gottgewollten Ordnung in allen Daseinsbereichen, die in Gott ihren Ursprung haben. Diese von Gott errichtete harmonische Ordnung stellt den Kern des scholastischen Ordo-Gedankens dar (Veit 1953). Diese Auffassung wurde vor allem durch Augustinus von Hippo und Thomas von Aquin entwickelt. ${ }^{3}$ Augustinus spricht von der „Zuteilung, die allen gleichen und ungleichen Dingen den ihnen eigenen Ort einräumt“". Seinerseits sieht Thomas im Kosmos die Einheit der Ordnung realisiert, nach der alle Geschöpfe auf Gott und aufeinander bezogen sind. Dieses Ordnungsprinzip gilt auch in Bezug auf die gesellschaftlichen Verhältnisse und demgemäß, wie Thomas sagt, sollen alle Bürger zur Regierung, zueinander und zu den Ausländern in geordneter Weise bezogen sein. ${ }^{5}$

Einer weiter verbreiteten Lesart zufolge führte das scholastische Ordo-Denken zu einer statischen Gesellschaftsauffassung. Der Scholastik wird vorgeworfen, sie habe die historisch gegebene Gesellschaftsordnung als gottgewollt legitimiert und den Harmonie- und Friedensgedanken des Ordo auf die Sozialstruktur übertragen. Die Verteilung der Einkommen sei entsprechend des standesgemäßen Bedarfs (der „Idee der Nahrung“) erfolgt. Dadurch seien sozialer Wandel und soziale Mobilität unterbunden worden, die mittelalterliche Gesellschaft sei ökonomisch und sozial erstarrt (Bücher 1925, S. 222-224; Sombart 1911, S. 141-144). Dieser Auffassung hat Joseph Höffner energisch in seiner wirtschaftswissenschaftlichen Dissertation mit dem Titel „Wirtschafsethik und Monopole im fünfzehnten und sechzehnten Jahrhundert" (Höffner 1941) ${ }^{6}$, die er 1940 bei Eucken in Freiburg abschloss, widersprochen.

Höffner verwirft die u.a. Anschauung über die mittelalterliche und frühneuzeitliche Gesellschaft als ungeschichtlich und verweist auf die hohe wirtschaftliche Dynamik während der commercial revolution. Er hat darüber hinaus die Thesen zurückgewiesen, dass die scholastischen und spätscholastischen Theologen dem Markt, dem Handel, dem Gewinnstreben und der freien Konkurrenz gegenüber feindlich gesinnt waren, dass sie eine statische und geschlossene Wirtschaftsgesinnung hatten und dass sie die Idee der auskömmlichen Nahrung und der standesgemäßen Versorgung und Lebensunterhalt vertreten haben. Die Scholastiker haben in der Tat die natürliche Marktfreiheit anerkannt. Sie bekunden „ein lebhaftes Interesse für die wirtschaftliche Umwelt, die sie weder moralisierend verwerfen, noch

3 Zur Begriffsgeschichte der Ordo-Idee ausphilosophischer und theologischer Perspektive vgl. Krings 1941/1982; Gässler $1950 / 1994$.

4 Augustinus von Hippo: De civitate dei, 19, 13: „Ordo est parium dispariumque rerum sua quique loca tribuens dispositio“.

5 Vgl. Thomas von Aquin: Summa Theologiae, I. II, q. 104, a. 4.

6 Zur Analyse der ökonomischen, politischen und sozialethischen Beiträgen Höffners und zur Beziehung zwischen seinen Auffassungen über die christliche Sozialethik und dem Ordoliberalismus vgl. Goldschmidt und Nothelle-Wildfeuer 2010; Franco 2015. 
vorbehaltlos bejahen, sondern mit kritischen Augen betrachten." (Höffner 1941, S. 170).

Der Begriff Ordo stellt „eines der ranghöchsten Symbolwerte - vielleicht das Ranghöchste - der scholastischen Metaphysik" (Veit 1953, S. 7) dar. Diese Idee drückt die Auffassung aus, dass es in allen Dingen eine bestimmte Beziehung und Interdependenz gibt. Auch Eucken, der sich hier explizit auf den scholastischen Ordo-Gedanken bezieht, charakterisiert inn als „sinnvolle Zusammenfügung des Mannigfaltigen zu einem Ganzen" (Eucken 2004, S. 372), obwohl er diesen Begriff nicht ausführlich behandelt. Die Auffassungen, die Eucken in "Die Grundlagen der Nationalökonomie" (Eucken 1950, S. 239-241) vertritt, finden sich nahezu wörtlich in "Grundsätze der Wirtschaftspolitik" (Eucken 2004, S. 372-374) wieder.

Eucken verwendet den Ordo-Begriff in zweifacher Weise. Einerseits bezeichnet er damit die empirisch existente „Wirtschaftsordnung“ als „eine konkrete, positiv gegebene Tatsache. Sie ist die Gesamtheit der realisierten Formen, in denen in concreto jeweils der alltägliche Wirtschaftsprozeß abläuft". Andererseits hat die Ordnung Eucken zufolge noch eine weitere Bedeutung "die dem Wesen des Menschen und der Sache entspricht; das heißt Ordnung, in der Maß und Gleichgewicht bestehen." (Eucken 2004, S. 372, 23, 180). Hier sieht Eucken eine dynamische Bedeutung des Begriffes Ordnung, die zu suchen und zu realisieren ist und die eine Gestaltungsaufgabe voraussetzt. Diese Ordnung muss nach Eucken nicht nur einen Rahmen besitzen, sondern auch vom Menschen gestaltet werden. ${ }^{7}$

Eucken unterscheidet zwischen den konkreten und den verwirklichten Ordnungen und derjenigen Form der Ordnung, die gerecht ist und die der Menschenwürde entspricht. Daraus folgt für Eucken, dass beide Begriffe unentbehrlich sind, d.h. die "Ordnungen als individuelle, wechselnde Tatbestände der Geschichte" und "Ordnung als Ordo" (Eucken 1950, S. 239; 2004, S. 373), die eine Ordnungs- und Gestaltungsaufgabe darstellen. Die Ordo-Idee besteht für Eucken in der Suche nach einer funktionsfähigen und menschenwürdigen Ordnung für die industrialisierte Wirtschaft: „Die Wirtschaftspolitik aber soll die freie natürliche gottgewollte Ordnung verwirklichen" (Eucken 2004, S. 176).

Eucken unterscheidet dadurch die konkreten Wirtschaftsordnungen von dem Streben nach Ordnung der Wirtschaft:

7 Eucken bezieht sich hierbei auf das Ordo-Verständnis von Augustinus und Thomas. Vgl. Eucken 1950, S. 239. Zum Bezug auf Augustinus und Thomas von Aquin vgl. auch Eucken 2004, S. 348, 372.
„Dieser Unterschied der Begriffe ist so alt, weil er in der Auseinandersetzung mit der konkreten Wirklichkeit fast notwendig entsteht. Der Mensch will wissen, wie die konkreten Ordnungen sind, und er sucht eine bessere Ordnung. Aber beide Begriffe kontrastieren miteinander: die konkreten, unbefriedigenden Ordnungen, in denen die Menschen faktisch leben, und die brauchbare und gerechte Ordnung." (Ebd., S. 373).

Eucken bezieht sich in seinen Überlegungen auch auf die Auffassungen der Physiokraten des 18. Jahrhunderts, die aus dem Naturrechtsdenken die natürlichen Gesetzmäßigkeiten der wirtschaftlichen Prozesse abzuleiten versuchten, indem sie zwischen einer natürlichen Ordnung oder ordre naturel als eine von Gott geschaffene Weltordnung und dem ordre positif als die vom Menschen gesetzte Ordnung unterscheiden. Dabei gingen sie von einer Vorstellung einer vorgegebenen Harmonie der Weltordnung aus. Eucken lehnt gleichwohl die Ansichten des Laissez-faire Liberalismus und der Physiokraten ab, welche von der Vorstellung einer prästabilierten Harmonie und von der Idee, dass die Wettbewerbsordnung und die menschenwürdige Ordnung aus sich selbst heraus entstehen können, getragen waren (Ebd., S. 53).

Im Unterschied zum klassischen Liberalismus plädiert Eucken für die Rolle der staatlichen Wirtschaftspolitik. Er betont vor allem die Rolle des Staates als Schiedsrichter, der der Entstehung wirtschaftlicher Machkonzentrationen vorbeugen soll, die die Realisierung der Wettbewerbsordnung verhindern. Der Staat hat die Aufgabe, eine gerechte Rahmenordnung und Spielregeln zu schaffen, die eine effiziente Wettbewerbsordnung gewährleisten und die Degenerationen des Wettbewerbs verhindern sollen.

Eucken greift die Kritik an dem klassischen Liberalismus auf und verteidigt die Notwendigkeit rechtlicher und politischer Voraussetzungen für die Ordnung des Wettbewerbs:

„Die Wettbewerbsordnung verwirklicht sich nicht von selbst. Sie ist in diesem Sinn keine natürliche Ordnung, kein ordre naturel. Es genügt nicht, gewisse Prinzipien des Rechtes zu verwirklichen und im Übrigen die Entwicklung der Wirtschaftsordnung sich selbst zu überlassen. Aber in einem anderen Sinne ist sie eine natürliche Ordnung oder Ordo. Sie bringt nämlich die starken Tendenzen zur Wirkung, die auch in der industriellen Wirtschaft zur vollständigen Konkurrenz drängen. Indem die Wirtschaftspolitik diese Tendenzen als Ordnungsformen wirksam macht, tut sie das, was der Natur der Sache und des Menschen entspricht. [...] 
Es zeigt sich, daß die Politik der Wettbewerbsordnung [...] von einer Gesamtentscheidung ausgeht und eine Wirtschaftsverfassung aufstellt, mit anderen Worten, daß sie eine Ordnung setzten will." (Eucken 2004, S. 373).

\section{Kritiken und Widerlegungen: Eine Würdigung der Ordo-Idee bei Eucken}

In der sekundären Literatur um das Ordo-Verständnis von Eucken sind die Interpretationen sehr unterschiedlich und aufgrund seiner wenigen Ausführungen missverständlich ${ }^{8}$, vor allem bezüglich der Aussage, der Ordo-Gedanke sei als eine freie natürliche, gottgewollte Ordnung aufzufassen. Der Ordoliberale Karl Paul Hensel behauptet, dass sich alle ordnungspolitischen Bemühungen auf „vorgegebene ,im Sein verankerte Möglichkeiten der Ordnung" (Hensel 1949, S. 267) konzentrieren sollten. In ähnlicher Weise drückt sich auch der Jurist und Ordoliberale Franz Böhm aus. Dieser betont, dass die Idee der prästabilierten Harmonie das ist, was Eucken unter dem Begriff Ordo versteht, d.h. ,eine vom Menschen vorgefundene, nicht von ihm geschaffene Ordnung" (Böhm 1950, S. XLVIII).

In der Diskussionen der letzten Jahren wird darüber hinaus einerseits Euckens metaphysische Legitmimierung des Ordo-Begriffes kritisiert (Voigt 1996, S. 160), andererseits wird auch die gegensätzliche Auffassung vertreten, dass genau in der fehlenden metaphysischen Fundierung von Euckens Auffassung der Grundstein für die mögliche Anschlussfähigkeit des ordoliberalen Konzeptes zu den modernen ökonomischen Theorien liegt (Pies 2001, S. 5-7) $)^{9}$ Von Seiten der katholischen Sozialethiker ist hier auf die Stellungnahme Oswald von Nell-Breunings hinzuweisen, der, obwohl er bemerkt, dass mit der Ordo-Idee der "subtheologische Gedanke der ,invisible hand" preisgegeben“ wird, den „rein formalen Charakter" (Nell-Breuning 1954, S. 218) der neoliberalen Utopie kritisiert.

Seinerseits sieht der Dominikaner Egon Edgar Nawroth - der in seiner massiven Kritik ein verfehltes Zerrbild des Ordoliberalismus und der Sozialen Marktwirtschaft gezeichnet hat - im Ordo-Gedanken des Ordoliberalismus einen Widerspruch zur scholastischen Tradition. Er versucht auch zu zeigen, dass den

8 Für einen Überblick über die verschiedenen Interpretationen von und Reaktionen auf Euckens Auffassungen vgl. Goldschmidt 2002, S. 101-108; Becker 1965, S. 89-98.

9 Für eine Kritik zu diesen Interpretationen vgl. Goldschmidt 2002, S. 102-105.
Ordo- und Neoliberalen ein Begriffsnominalismus und eine individualistisch-funktionaltheoretische Entleerung des Ordo-Begriffs zu eigen ist, weil dieser Begriff „im unbewiesenen Dogma von der Naturordnung des Wettbewerbs" (Nawroth 1961, S. 383) liegt und sich auf die Idee stützt, dass nur die Wirtschaftsordnung in der Lage sei, eine effiziente und menschenwürdige Ordnung zu schaffen. Nawroth führt aus, dass Euckens Ordo-Idee und Ordnungskonzeption, obwohl er sich ausdrücklich auf die scholastische Tradition des Ordo-Begriffes bezieht, eine „wirtschaftsimmanente" und „kausalmechanische" Prägung (Ebd., S. 385) hat, die nicht auf das Gemeinwohl ausgerichtet ist.

Dieser Auffassung setzt Nawroth die „Architektonik des thomistischen Ordnungsbildes“ entgegen, „die jede Einzelaktion ebenso wie alle Kultursachbereiche in das Ordnungsgefüge der theozentrisch determinierten sittlichen Gesamtordnung eingefügt wissen will" (Ebd., S. 385). Den Ordoliberalen dagegen bleibe nur eine individualistische Auffassung des Ordo-Begriffes, verstanden als "eine leere Gleichgewichtsformel“ und ein rein funktionaler Begriff: „An die Stelle des deistischen Glaubens an die providentielle Wirtschaftsharmonie tritt der säkularisierte Glaube an die Gleichgewichtstendenzen des Marktmechanismus" (Ebd., S. 386).

Außerdem glaubt Nawroth, die Ordoliberalen seien "überzeugt" davon, dass die reine Verwendung der Begriffe der Gerechtigkeit und des Gemeinwohls genüge, um „die geistige Verbindungslinie“ (Ebd., S. 14) zu Thomas von Aquin und zur katholischen Soziallehre aufgewiesen zu haben. Tatsächlich jedoch weisen seiner Ansicht nach diese Begriffe bei den Ordoliberalen nur einen nominalistischen Charakter und deshalb eine negative Konnotation und Tragweite auf. Gemäß seiner Sicht leiden solche Begriffe im ordoliberalen Verständnis unter einer inhaltlichen Entleerung. Damit fehle innen der Wirklichkeits- und der Wesensbezug, und sie verlieren "den Anspruch auf Verbindlichkeit“ (Ebd., S. $45)^{10}$ Mit anderen Worten, wie Ernst-Wolfram Dürr sagt, steht Euckens Ordo-Begriff im Gegensatz zu dem der Scholastik (Dürr 1954, S. 165).

Gegen diese Missverständnisse ist einzuwenden, dass Eucken sowohl eine metaphysische Legitimierung des Ordo-Begriffes als auch seine ökonomische Ausrichtung im Blick hatte. Er betont ausdrücklich die metaphysische Dimension des Ordo-Gedankens. Dies bezeugt er in einem Brief aus dem Jahr 1943 an Alexander Rüstow: „Nicht dadurch verfiel m.E. der Liberalismus,

10 Für eine kritische Würdigung der Auffassungen von Nawroth im Lichte der Sozialen Markwirtschaft von Wilhelm Röpke vgl. Franco 2016, S. 293-318. 
daß er religiös-metaphysisch fundiert war. Im Gegenteil. Sobald er seinen religiös-metaphysischen Gehalt verlor, verfiel er - was sich nun ganz genau historisch und systematisch erweisen läßt."11 In der Tat lehnt Eucken, wie Nils Goldschmidt in seiner Untersuchung gezeigt hat, nur den Laissez-faire Liberalismus ab, sein HarmonieDenken und dessen wirtschaftspolitische Konzeption, jedoch nicht die Intention und die Suche nach der „freien natürlichen gottgewollten Ordnung" (Goldschmidt 2002, S. 105). Diese Idee kommt deutlich im folgenden Zitat zum Ausdruck: „Der Wirtschaftspolitik des Laissez-faire lag ein großer Gedanke zugrunde. Freiheit soll gegeben werden, damit sich die natürliche, gottgewollte Ordnung entwickelt“. Eucken fügt dennoch hinzu, dass diese Wirtschaftspolitik „nicht das erreichte, was sie wollte“: „Und auch eine freie, natürliche Ordnung entsteht nicht einfach dadurch, daß die Wirtschaftspolitik ihre Verwirklichung der Entwicklung überläßt - sondern nur dann, wenn sie selbst darauf gerichtet [ist]." (Eucken 2004, S. 53).

\section{Der christliche Glaube als ethische Grundlage des Ordoliberalismus von Eucken}

Eine weitere umstrittene Frage bei der Diskussion von Euckens Auffassungen betrifft den Wert, den der christliche Glaube für sein gesamtes Denken annimmt. Es handelt sich hierbei um einen Aspekt, den die wissenschaftliche Diskussion oft übersehen oder nicht angemessen berücksichtigt hat. In der neuen Forschung wurde das Thema jedoch positiv gewürdigt. Aus der Analyse von Euckens Auffassung ergibt sich, dass sein Hinweis auf den christlichen Glauben in Verbindung mit den normativen Grundlagen der politischen Ökonomie und der Verfassung der ordoliberalen Wirtschafts- und Gesellschaftsordnung gebracht werden soll (Goldschmidt 2002, S. 121-136; 1998; Müller 2007; Nass und Müller 2013).

\subsection{Die Freiburger Kreise und der Widerstand gegen den Nationalsozialismus}

Die christliche und ethische Grundlage des wissenschaftlichen Wirtschaftsprogramms Euckens hat ihre Wurzel in seinem biographischen und intellektuellen Weg, der im Zusammenhang mit der historischen Situation der Krise des Liberalismus und des Kapitalismus sowie mit der Zeit des ersten Weltkrieges und des

11 Brief von Eucken an Rüstow, zitiert nach Lenel 1991, S. 13.
Aufstiegs des Nationalsozialismus steht. Die Bedeutung der christlich-ethischen Idee und die Beziehung zwischen wissenschaftlicher Analyse und ethischer Reflexion bei Eucken wird deutlich anhand seiner Beteiligung an der Denkschrift des sogenannten Freiburger Bonhoeffer-Kreises. Dieses Dokument wurde in den Jahren 1942/1943 auf Einladung des Theologen und lutherischen Pastors Dietrich Bonhoeffer verfasst und blieb bis zum Jahr 1979 unveröffentlicht.

Bevor die ethische und normative Relevanz der Inhalte der Denkschrift im Fokus steht, sollen zunächst schematisch die drei Freiburger Kreise dargestellt werden, die sich während der Jahre 1938 bis 1944 gebildet haben und deren kulturelles Epizentrum an der Universität Freiburg lag. Zu den Mitgliedern dieser Kreise gehörten Vertreter der katholischen und protestantischen Kirchen sowie Wissenschaftler und Professoren, die entweder in Freiburg tätig waren oder in engerem Kontakt zu der dortigen Universität standen. Die Mitglieder dieser Kreise trafen sich regelmäßig und meistens heimlich, um über die damalige wirtschaftliche, politische und soziale Lage zu diskutieren. Diese Kreise bildeten die intellektuelle Stimme des ideologischen Dissens zu und des Widerstandes gegen den Nationalsozialismus. Sie spielten darüber hinaus eine wichtige Rolle bei der theoretischen Ausarbeitung der Wirtschafts- und Gesellschaftsordnung, die nach dem Zweiten Weltkrieg verwirklicht werden sollte. ${ }^{12}$ Sie stellten außerdem eine intellektuelle Kritik gegen Martin Heidegger dar, der in Jahr 1933/1934 das Rektorat der Universität Freiburg übernahm und deutliche Sympathie für den Nationalsozialismus erklärte. ${ }^{13}$

Der zeitlich erste Widerstandszirkel wurde auf Initiative der Ökonomen Constantin von Dietze und Adolf Lampe als Reaktion auf die sogenannte Reichskristallnacht vom 10./11. November 1938 und die Zerstörung der Synagoge von Freiburg konstituiert. Dieser Kreis wurde als Freiburger Konzil bezeichnet. Unter seinen führenden Mitgliedern soll vor allem der Historiker Gerhard Ritter hervorgehoben werden, der einen besonderen Einfluss auf die weiteren Freiburger Kreise und Widerstandszirkel ausgeübt hat. Darüber hinaus gehörten zum Kreis der Kirchenhistoriker Clemens Bauer, der Ordoliberale Franz Böhm und Vertreter der Katholischen Kirche und der Bekennenden Kirche, eine

12 Zur Rekonstruktion und zum Überblick über diese Kreise vgl. Goldschmidt 2008, S. 91-96; Goldschmidt 2002, S. 124-129; Aus diesen Texten sind auch die Darstellung und Ausführungen über die Freiburger Kreise entnommen.

13 Zur Widerstandsrolle der Freiburger Kreise gegen den Nationalismus im universitären Milieu und gegen Heidegger vgl. Goldschmidt 2005a; Secci 1998. 
Oppositionsbewegung innerhalb der evangelischen Kirche gegen den Nationalsozialismus. Im Jahr 1938 wurde von einigen Mitgliedern des Freiburger Konzils, größtenteils von Ritter, eine Denkschrift mit dem Titel „Kirche und Welt - Eine notwendige Besinnung auf die Aufgaben des Christen und der Kirche" verfasst (Schwabe und Reichardt 1984, S. 635-654).

Ein zweiter Freiburger Kreis wurde durch die Arbeitsgemeinschaft von Beckerath gebildet, die nach dem Nationalökonom Erwin von Beckerath benannt wurde. Diese Arbeitsgemeinschaft, die nicht direkt in Freiburg entstanden ist, hatte ihren Ursprung in der Klasse IV der Akademie für deutsches Recht in Berlin, aus der die Arbeitsgemeinschaft Volkswirtschaftslehre unter der Leitung von von Beckerath entstand. In diesem Arbeitskreis wurde im Jahr 1941 ein Unterausschuss eingerichtet, zu dem Dietze, Lampe, Eucken und Bauer gehörten. Die Arbeitsgemeinschaft Volkswirtschaftslehre wurde im Jahr 1943 aufgelöst, dennoch trafen sich ihre Mitglieder bis zum Jahr 1944 weiter und verfassten verschiedenen Texte und Protokolle (Blumenberg-Lampe 1986).

Der sogenannte Freiburger Bonhoeffer-Kreis entwickelte sich auf die Anregung des Berliner Pastors Dietrich Bonhoeffer hin, der zusammen mit der Bekennenden Kirche den Arbeiten von Ritter und Dietze besondere Aufmerksamkeit und starkes Interesse schenkte. Bonhoeffer erteilte einigen Freiburger Professoren die Bitte und zugleich den Auftrag, eine neue Programmschrift zu verfassen, die die Grundsätze und Prinzipien für eine Rekonstruktion einer auf den Grundlagen der christlichen Ethik und des christlichen Glaubens beruhenden gerechten Wirtschafts- und Gesellschaftsordnung darlegen sollte.

Aus den verfügbaren Quellen ist es nicht möglich, vollständig zu rekonstruieren, warum Bonhoeffer diese deutschen Wissenschaftler damit beauftragte, diese Programmschrift zu verfassen. Man kann jedenfalls feststellen, dass die Arbeiten des Freiburger Konzils, die Beiträge von Dietze und Ritter sowie ihr Widerstand gegen den Nationalsozialismus der Bekennenden Kirche und Bonhoeffer selbst bekannt waren. Bonhoeffer nahm auch persönlich Kontakt zu Dietze und Ritter auf. Der Einladung von Bonhoeffer folgend involvierte Dietze einige Mitglieder des Freiburger Konzils und gründete einen neuen Arbeitskreis, dem auch Eucken, Lampe und Ritter angehörten. Es folgten mehrere Treffen in Freiburg, in denen die von Ritter vorbereitete Vorlage des Haupttextes der Denkschrift diskutiert wurde. Es fanden auch weitere Geheimtreffen in Freiburg zwischen dem 17. und 19. November 1942 statt, bei denen auch die Teilnahme von Bonhoeffer vorgesehen war. Die endgültige Ausarbeitung dieser neuen Denkschrift wurde im Januar 1943 fertiggestellt.

Der Text erhielt den Titel „Politische Gemeinschaftsordnung" und wurde mit dem Untertitel „Ein Versuch zur Selbstbesinnung des christlichen Gewissens in den politischen Nöten unserer Zeit" versehen. Diesem Dokument, das neben einem Vorwort aus zwei Hauptteilen besteht, wurden fünf Anlagen hinzugefügt. Unter den renommierten Autoren und Mitverfassern der Denkschrift bzw. der Anlagen sind vor allem der Historiker Ritter, die Ökonomen Dietze, Lampe und Eucken, die Juristen Erik Wolf und Franz Böhm sowie die Theologen Otto Dibelius und Helmut Thielicke zu erwähnen. Im ersten Hauptteil wird die Analyse der damaligen gesellschaftlichen, politischen und wirtschaftlichen Zusammenhänge angeboten. Der zweite Hauptteil - mit dem Titel „Grundzüge einer politischen Gemeinschaftsordnung nach christlichen Verständnis" - behandelt Fragen der christlichen Ethik sowie die praktischen Forderungen für die Realisierung einer neuen Gesellschaftsordnung. Den beiden Hauptteilen folgen fünf Anlagen, die jeweils die folgenden Titel tragen: Erstens „Rechtsordnung“, zweitens „Kirchenpolitik“, drittens „Erziehung“, viertens „Wirtschaft" sowie fünftens „Judenfrage“.

Die Denkschrift und die Anlage blieben bis zum Jahr 1979 unveröffentlicht (In der Stunde Null 1979). Nach dem gescheiterten Attentatsversuch auf Hitler vom 20. Juli 1944 gelang ein Teil der Denkschrift in die Hände der Gestapo. Dietze, Lampe und Ritter wurden inhaftiert und kamen nach Kriegsende wieder frei. Eucken dagegen wurde zwar verhört, aber nicht verhaftet, weil es nicht gelang, ihm die Beteiligung an der Niederschrift der Denkschrift zuzuschreiben.

\subsection{Die Wirtschafts- und Gesellschaftsordnung nach der Anlage 4 der Denkschrift des Freiburger Bonhoeffer-Kreises}

Die Anlage 4 der Denkschrift trägt der Titel „Wirtschaftsund Sozialordnung" und stellt eine Abhandlung dar, die von Dietze, Eucken und Lampe verantwortet wurde (Ebd., S. 128-145). Dietze trug die Hauptverantwortung bei der Verfassung dieser Anlage. ${ }^{14}$ Tatsächlich finden sich in der Anlage 4 wichtige Bezüge zur Ordo-Idee. Diese Anlage bietet darüber hinaus eingehend die Aus-

14 Im Folgenden werden vor allem die Auffassungen der Anlage 4 der Denkschrift des Freiburger Bonhoeffer-Kreises herangezogen. Die Behandlung der gesamten Denkschrift würde den Rahmen dieses Beitrages springen. Für eine ausführlichere Analyse der Struktur, Inhaltes und Bedeutung der Denkschrift und ihre Teile siehe die grundlegende Studie von Holthaus 2015. 
führung der wirtschafts- und sozialpolitischen Grundideen für die Gestaltung einer gerechten Wirtschaft- und Gesellschaftsordnung nach dem Krieg (Holthaus 2015, S. 239-269). ${ }^{15}$ Des Weiteren bilden die dort enthaltenen Auffassungen zusammengefasst die grundlegenden Prinzipien des Ordoliberalismus Euckens bzw. der späteren sozialen Markwirtschaft.

Das Ziel, das Dietze, Eucken und Lampe mit der Anlage 4 verfolgten, bestand mehr in der Ausarbeitung einer Gesamtordnung des Wirtschaftslebens als in „den Pflichten und Geboten, die nach christlicher Lehre für das Verhalten des einzelnen Menschen im Wirtschaftsleben gelten." (In der Stunde Null, S. 128). Obwohl die drei Verfasser der Denkschrift-Anlage die Bedeutung der Wirtschaftsethik als Individualethik anerkannten, ging es innen vor allem darum, „die Grundlagen der Sozial-Wirtschaftsethik christlich zu begründen, gerade nach evangelischem Verständnis" (Ebd.). Ihre Aufgabe war es, das Programm der zukünftigen Wirtschafts- und Gesellschaftsordnung so zu formulieren, dass es an den Prinzipien des Evangeliums und der christlichen Ethik orientiert sein sollte.

$\mathrm{Zu}$ diesem Zweck haben sich die Autoren von folgenden Kriterien und Grundsätzen leiten lassen:

\section{„I. Richtschnuren und Verbote, die sich nach unserem Glauben aus Gottes Wort für die Wirtschaft und ihre Ordnung ergeben, die also die Kirche vertreten kann und muß; II. Grundsätze, die sich aus Sachnotwendigkeiten des Wirtschaftens ergeben und die für seine Ordnung dauernde Geltung besitzen; III. eine sachliche Würdigung der gegenwärtigen und der nach menschlicher Voraussicht bevorstehenden wirtschaftlichen Lage." (Ebd.).}

Die Redakteure teilen vollständig die Ansicht, dass es innen nicht darum geht, eine „besondere evangelische oder auch nur allgemein-christliche Wirtschaftsordnung“ (Ebd.) zu entfalten. Sie argumentieren dafür, dass man „nicht aus den Grundlagen unseres Glaubens für die Wirtschaftsordnung genaue Regelungen mit dem Anspruch auf unverbrüchliche Gültigkeit ableiten" (Ebd., S. 128) könne. Sie betonen, dass es die Aufgabe der menschlichen Vernunft sei, die Problemlage der Zeit zu untersuchen und angemessene Lösungen anzubieten. Ihre Aufgabe folgt einem ethischen und sozialen Ziel und orientiert sich an der öffentlichen Rolle der Kirche und der christlichen Konfessionen:

15 Bereits im zweiten Hauptteil der Denkschrift befindet sich die Behandlung von wirtschafts- und sozialpolitischen Fragen (Holthaus 2015, S. 74-82).
"Worauf es uns ankommen muß, ist, eine Wirtschaftsordnung vorzuschlagen, die - neben ihren sachlichen Zweckmäßigkeiten - den denkbar stärksten Widerstand gegen die Macht der Sünde ermöglicht, in der die Kirche Raum für ihre eigentlichen Aufgaben behält und es den Wirtschaftenden nicht unmöglich gemacht oder systematisch erschwert wird, ein Leben evangelischer Christen zu führen." (Ebd., S. 128 f.).

Die Beschäftigung mit und die Stellungnahme der Kirche zu wirtschaftlichen Fragen wird dadurch gerechtfertigt, dass die christlichen ethischen Prinzipien und Gebote, die in der Nächstenliebe, in der Verteidigung des Eigentumsrechts und den menschlichen Arbeitsbedingungen zum Ausdruck kommen, nicht nur für den Bereich der Individualethik, sondern auch für das Wirtschaftsleben im Allgemeinen und in den verschiedenen Ordnungen gelten. Das Fundament der Weltverantwortung der Kirche und ihr Engagement in wirtschaftlichen Fragen wird theologisch oder besser gesagt christologisch gesehen, wie es die folgende Formulierung der Anlage bezeugt: „In ihrer Stellungnahme zur Wirtschaftsordnung muß die Kirche von Christus zeugen. Sie dient damit der Befreiung von allen weltlichen Heilslehren." (Ebd., S. 129).

Aus diesem Bewusstsein her stammt auch die Interpretation des Dekalogs der zehn Gebote nicht in einem engeren juristischen Sinn, sondern als Ausdruck der Anforderungen für die Wirtschaftsordnung. Die zehn Gebote bilden darüber hinaus die Grundlage der Stellungnahme der evangelischen Kirche zur Wirtschaftsfragen. Die konkreten Anforderungen, die der Dekalog mit sich bringt, bestehen darin, dass die „Vergötzung irdischer Güter und Mächte“ (Ebd., S. 129) vermieden werden und dass die Wirtschaftsordnung der „Gültigkeit gegenüber dem Namen und dem Worte Gottes Vorschub leisten“ (Ebd.) muss. Die Wirtschaft soll darüber hinaus den Menschen als sittliche Person achten und die Achtung der natürlichen Gemeinschaft ermöglichen und fordern. Die Kirche hat außerdem die Aufgabe, Grenzen abzustecken, Verbote zu verkündigen sowie „einige feste Richtschnuren für den Inhalt der Wirtschaftsordnung“ zu geben: „Die Kirche hat jedoch keinen Auftrag und keine Machtvollkommenheit, für die Ausgestaltung der Wirtschaftsordnung im Einzelnen ständig verbindliche Lösungen anzubieten. [...] Was die Kirche nicht selbst zur Wirtschaftsordnung zu sagen berufen ist, hat sie den christlichen Laien zu überlassen" (Ebd., S. 130), die sich um die konkrete Gestaltung einer gerechten Wirtschaftsordnung bemühen sollen.

Ein weiterer Aspekt, den das Dokument betont, ist die Überzeugung, dass die Wirtschaft den Menschen zu 
dienen hat: „Mit materiellen Kräften allein läßt sich das menschliche Leben nicht erträglich gestalten" und die Volkswirtschaft „bedarf der gesicherten Rechtsordnung und der festen sittlichen Grundlage" (In der Stunde Null, S. 131) zur Beachtung der Menschenwürde und des Wertes des Menschen. Mit anderen Worten stellen die Redakteure fest, dass jede Wirtschaftsordnung „bestimmter politischer und sittlicher Voraussetzungen bedarf" (Ebd., S. 134).

Die ethischen Prämissen, die im Vorwort und im ersten Teil der Anlage enthalten sind, wurden schließlich auf die folgenden Ausführungen über die gesellschaftlichen und politisch-ökonomischen Probleme angewendet, mit denen sich der Text in den weiteren Teilen auseinandersetzt. Die Verfasser stellen fest, dass die Sozialpolitik das Ziel verfolgen muss, „den Aufbau einer rechten Societas" anzuleiten. Die Sozialpolitik muss sich nicht „auf zusammenhanglose Fürsorgemaßnahmen beschränken; sie muß die gesamte Societas festigen und ständig im Einklange mit den Grundsätzen der Gesamtwirtschaftsordnung stehen." (Ebd., S. 132). Die Anlage 4 führt aus, das die Wirtschaftsordnung eine soziale Ordnung braucht, weil keine Wirtschaftsordnung von sich aus die soziale Ziele gewährleisten und realisieren kann: „Die Societas kann nicht lediglich nach wirtschaftlichen Gesichtspunkten geregelt werden" (Ebd., S. 132).

Diese Auffassung von Sozialpolitik unterstreicht die Idee der Interdependenz der Ordnungen und die enge Verbindung, die Eucken in seinen Hauptwerken weiterentfaltet hat, zwischen Sozialpolitik, Wirtschaftspolitik und Wirtschaftsordnung. Sie zeigt die Beziehung zwischen der notwendigen ethischen Reflexion und der sachlichen Wissenschaftsanalyse. Mit Recht stellt Leonhard Miksch in Bezug auf Euckens Gedankenwelt fest: „Erst nach strenger Durchführung des Denkprozesses werden sie [Rationales und Emotionales] wieder zur normativen Forderung verschmolzen. Religiöse, weltanschauliche Elemente ergänzen sich so widerspruchfrei mit nüchterner theoretischer Untersuchung." (Miksch 1937, S. 24)

Die Anlage 4 enthält darüber hinaus - wie bereits angedeutet - die konkreten wirtschaftspolitischen und sozialpolitischen Leitlinien für die Gestaltung der Nachkriegsordnung. Es geht um Anforderungen und Aspekte, die mit den Grundlagen der späteren Sozialen Marktwirtschaft übereinstimmen. Die Anlage würdigt positiv eine auf dem Wettbewerb basierende Marktwirtschaft, ohne sie zu verherrlichen. Die Anlage betont auch die Notwendigkeiten der ,politischen und sittlichen Voraussetzungen“ (In der Stunde Null, S. 134), auf die eine gerechte Wirtschaftsordnung beruhen soll.
Darüber hinaus plädiert sie für eine Ordnung des Wettbewerbs, nämlich für einen "geordneten Wettbewerb" (Ebd., S. 131).

Ein zentraler Aspekt der Denkschrift ist einerseits die Forderung nach einem freien Wettbewerb und andererseits nach der Notwendigkeit des sozialen Ausgleichs. Die Denkschrift kritisiert auch im Sinne Euckens die Monopolbildungen und setzt sich für die Verhinderung von Kartellen ein. In der Denkschrift findet auch das katholische Sozialprinzip der Subsidiarität Berücksichtigung. Die Denkschrift plädiert in der Wirtschaftspolitik für „selbständige Initiative und Freiheit der Wirtschaftenden“ (Ebd., S. 91), sowie für „kleine Einheiten“ und Gemeinschaften (Ebd., S. 87 f., 141). Das Dokument weist auch auf die Bedeutung der Sozialversicherungen im Zusammenhang mit der Selbstverantwortung hin: „Alle staatliche Sozialfürsorge kann zur Gefahr werden, wenn sie der Verantwortungsfreudigkeit der Wirtschaftenden Abbruch tut" (Ebd., S. 90).

Nils Goldschmidt fasst mit drei wesentlichen Aspekten die ethischen Grundlinien zusammen, die die Ausarbeitung und die Absicht der Anlage 4 geleitet und geprägt haben:

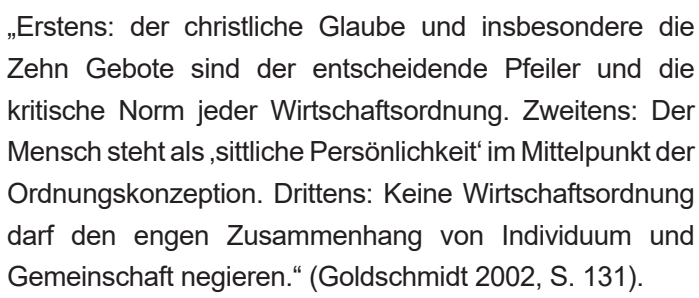

Der Vergleich zwischen den ethischen Voraussetzungen und Prinzipien, die in dieser Anlage enthalten sind, und den ordnungspolitischen Ansichten, die Eucken in seinem Buch "Grundsätze der Wirtschaftspolitik" formuliert hat (vgl. Goldschmidt 2002, S. 131-136; 1998; 2008, S. 95-96), weist eine Entsprechung und Korrespondenz nach. ${ }^{16}$ Obwohl Euckens Beteiligung an der Verfassung

16 Vgl. Goldschmidt 2002, S. 135: „Verläßt man nun die Detailüberlegungen, läßt sich zusammenfassend sagen: Die Denkschrift-Anlage entspricht insbesondere in ihrer konzeptionellen Herangehensweise weitestgehend dem ordoliberalen Programm, wie es Walter Eucken in den ,Grundsätzen der Wirtschaftspolitik ausformuliert hat. Im Mittelpunkt steht die Idee der Ordnung, wobei - entsprechend den unterschiedlichen Herangehensweisen - die Denkschrift eher den Bezug zum christlichen Verständnis sucht, Eucken in den ,Grundsätzen“ den Ordnungsbegriff - zwar nicht weniger normativ - jedoch eher methodisch einsetzt. In Einzelfragen lassen sich zwar Unterschiede aufweisen, die aber - nicht zuletzt da eine Denkschrift immer nur fragmentarisch bleiben kann - für die grundlegende Herangehensweise keine Bedeutung haben.“ 
der Anlage nicht federführend war, kann man nicht bestreiten, dass sich Euckens ordoliberale Grundkonzeption im Dokument der Denkschrift und vor allem in der Anlage 4 wiederfindet: „Euckens ordoliberales Konzept der Wettbewerbsordnung war die wirtschaftspolitische Basis der Freiburger Denkschrift." (Holthaus 2015, S. 110).

\subsection{Der normative Gehalt des Ordo- Gedankens und die christliche Sozialethik}

Die von Dietze, Lampe und Eucken verfasste Anlage 4 der Denkschrift enthält bestimmte bedeutungsvolle Stellen, die das normative Fundament des ordnungsökonomischen und ordnungspolitischen Programms Eucken kennzeichnen. Es tauchen auch einige Aspekte auf, die den Auftrag und die Zuständigkeit der Kirche, zur Wirtschaftsordnung Stellung zu nehmen, und indirekt die Natur und Aufgabe der christlichen Sozialethik betreffen. Dabei wird auch die Notwendigkeit einer moralischen Einbettung der Wirtschaftsordnung betont.

In der Anlage 4 der Denkschrift des Freiburger Bonhoeffer-Kreises wird exemplarisch deutlich, dass die Wirtschafts- und Sozialpolitik des Ordoliberalismus „in ein normatives Grundgerüst eingebunden ist“ (Goldschmidt 2002, S. 128). Von daher können das Fundament und der Einfluss des christlichen Glaubens auf das Wirtschaftsprogramm, das Eucken in den Grundsätzen der Wirtschaftspolitik formuliert hat, „,vor diesem Hintergrund adäquat rekonstruiert werden" (Ebd., S. 122).

Der Ordo-Begriff stellt eine der Schlüsselkategorien der programmatischen Freiburger Denkschrift dar (Holthaus 2015, S. 67, 194-206). Vor allem in der Anlage 4 finden sich grundlegende Überlegungen, die mit Euckens wirtschaftspolitischer und ordnungsökonomischer Konzeption übereinstimmen. Zentral sind in der gesamten Denkschrift die Betonung der Schaffung von Ordnungsstrukturen und Regeln sowie die Einbettung der menschlichen und wirtschaftlichen Freiheit in eine höhere Rahmenordnung. Diese Idee betreffen auch die Aufgabe des Staates, den die Denkschrift im Sinne von Euckens Auffassung des "starken Staates“ als eine „Ordnungsmacht“ (In der Stunde Null, S. 68, 71, 93) betrachtet. Die Verwendung der „Ordnungen“ wird in der Anlage 4 auf verschiedene Bereichen angewendet, wie z.B. die Sozialordnung, die Wirtschaftsordnung, den Arbeitsschutz, die Haushaltspolitik und die Rechtsordnung. Von besonderer Bedeutung sind die Reflexionen über die Aufgabe des Staates und sein Verhältnis zur Wirtschaft. Der Staat soll nach der Denkschrift die passenden Rahmenordnungen und "geeignete Bedingungen" für die Wirtschaft (Ebd., S. 140) gestalten. Dennoch wird in der Denkschrift diese Ordnungsaufgabe des Staates im Sinne von Euckens Ordoliberalismus verstanden. Der Staat habe die Aufgabe, die Wirtschaft zu gestalten, aber nicht zu lenken (Ebd., S. 137 f.; Eucken 2004, S. 336). Mit anderen Worten betont die Anlage 4, dass „[e]ine auf weiteres Vorantreiben zentraler Leitung gerichtete Wirtschaftspolitik [...] abzulehnen [ist]" (In der Stunde Null, S. 137).

Die christlich-normative Grundlage prägte die Essenz des ordoliberalen Denkens von Eucken. In der Sekundärliteratur wird die Beurteilung der Frage nach dem persönlichen Glauben und nach der religiösen Prägung Walter Euckens kontrovers umstritten (Goldschmidt 2005b, S. 305-306; Holthaus 2015, S. 107-112; Oswalt 2005, S. 304). Sicher war Eucken weniger als die weiteren Verfasser der Freiburger Denkschrift mit kirchlichen Institutionen verbunden. Dennoch war er in Kontakt mit den Vertretern der Bekennenden Kirche und wurde auch als deren Ratgeber eingeladen.

Euckens Anerkennung für und seine Beschäftigung mit dem christlichen Glauben sowie seine aktive Beteiligung an der Bekenntniskirche und sein Widerstand gegen den Nationalsozialismus werden auch durch die Korrespondenz mit seiner Mutter Irene in den 30er und 40 er Jahren des letzten Jahrhunderts bezeugt. Dabei ergibt sich nicht nur sein persönliches Glaubensbekenntnis, sondern auch die Tatsache, dass der christliche Glaube für Eucken der Grund und der ethische Antrieb ist, um sein wirtschaftspolitisches Programm zu erarbeiten. Darüber hinaus spielen bei Eucken die Prinzipien des christlichen Glaubens eine theoretische Rolle und stellen eine normative Orientierung für seinen Zugang zur Wirklichkeit sowie für seine Analyse und Lösung der ökonomischen und gesellschaftlichen Probleme dar. ${ }^{17}$

In einem Brief vom 1942 an seinem Freund Rüstow schreibt Eucken: „Ich aber könnte weder existieren noch arbeiten, wenn ich nicht wüßte, daß Gott existiert." (Lenel 1991, S. 13). In einem Artikel aus dem Jahr 1932, in dem Eucken auf die Beziehung zwischen Religion, Wirtschaft und Staat eingeht, betont er, dass die Suche nach einer stabilen und gerechten Wirtschaftsund Gesellschaftsordnung nur von der Religion und im Glauben an Gott mit Erfolg durchgeführt werden kann und insofern sich der einzelne Mensch seiner Verankerung im Glauben bewusst wird:

17 Vgl. Goldschmidt 2002, S. 121-123. Hier werden einige Briefe von Eucken an seine Mutter Irene wiedergegeben und gewürdigt. 
„Die geschichtliche Entwicklung wird nach Scheitern aller anderen Versuche mit Notwendigkeit zu dem Ergebnis führen müssen, daß der umfassende Sinnzusammenhang den Tätigkeiten des einzelnen Menschen nur von der Religion, vom Glauben an Gott wieder verliehen werden kann. Erst dann wird auch auf sozialen und politischen Gebiet wieder eine gewisse Beruhigung eintreten.“ (Eucken 1932, S. 87).

Diese Überlegungen lassen sich im Lichte des OrdoGedankens zusammen mit ihrer ökonomischen, religiösen, metaphysischen und normativen Dimension interpretieren. Im Anhang zu den „Grundsätzen der Wirtschaftspolitik" greift Eucken die im Text der Denkschrift enthaltenen Themen wieder auf und setzt sich mit der christlichen Sozialethik und der katholischen Soziallehre auseinander. Er stellt fest, dass das Denken in Ordnungen des Ordoliberalismus aus der echten christlichen Tradition hervorgekommen ist, die sich bereits bei Thomas von Aquin und später bei den Sozialenzykliken findet. Eucken ist der Ansicht, dass die katholische und evangelische Kirche als ordnende Faktoren des wirtschaftlichen und gesellschaftlichen Lebens zu betrachten sind.

Für Eucken ist es notwendig, dass sich die Kirchen mit wirtschaftlichen Fragen auseinandersetzen. Obwohl sich die Kirchen „aus dem Streit der politischen Parteien heraushalten müssen[...] so kann es ihnen anderseits nicht gleichgültig sein, in welchen Ordnungen die ihnen anvertrauten Menschen leben." (Eucken 2004, S. 347). Er weist damit darauf hin, dass die Soziale Marktwirtschaft den Prinzipien der christlichen Sozialethik entspricht und dass die Kirchen Stellung beziehen müssen, wenn bestimmte Ordnungen "die Bedingungen der sittlichen und religiösen Existenz gefährden." Schließlich behauptet Eucken, dass das Subsidiaritätsprinzip „oberstes Prinzip für die Gestaltung des sozialen Lebens“ (Ebd., S. 347-350, hier 348) sei. Dennoch kann dieses Prinzip nur mit der Wettbewerbsordnung und nicht mit der berufsständischen Ordnung, die die Enzyklika „Quadragesimo anno“ vorgeschlagen hat, kompatibel sein.

Die Verbindung und Übereinstimmung zwischen dem scholastischen Ordo-Begriff und dem OrdoGedanken des Ordoliberalismus wird darüber hinaus von Müller-Armack bestätigt. In einer Stellungnahme, die auf Höffner Bezug nimmt, behauptet er, dass die theologische Schöpfungsordnung, „den Gedanken der Personalität und den des Ordo als gemeinsames christliches Fundament der Sozialtheorie" (Müller-Armack 1959, S. 565) anzuerkennen bestrebt sein sollte. In Anschluss an Höffner zieht Müller-Armack eine
Parallele zwischen dem Prinzip der freien Ordnung der Scholastik und den Auffassungen der ökonomischen Theorie der Sozialen Markwirtschaft, die „die Prinzipien eines freien Wettbewerbs als gesellschaftsnützliche Ordnung in einem sozialen Sinne begriffen" habe. Er lobt die Auffassungen der katholischen Soziallehre, die durch den Ordo-Gedanken „eine überlegene Weite“ gewinnt und der „modernen Sozialtheorie jenes feste und gleichzeitig der Konkretisierung zugängliche Gefüge mitteilt, dessen sie so sehr bedarf" (Ebd., S. 568). Müller-Armack interpretiert darüber hinaus das Konzept des Ordo-Jahrbuchs in dieser Richtung des scholastischen Denkens.

Die christliche und normative Grundlage des ordoliberalen Denkens und die Affinität zur christlichen Sozialethik wurden in der neuen wissenschaftlichen Diskussion positiv aufgenommen. Auf diesen Zusammenhang geht Christian Müller ein und behauptet, dass die ordoliberalen und neoliberalen Denker die Freiheit nicht als den höchsten Wert in ihren wirtschaftlichen und sozialen Auffassungen betrachtet haben. Im Zentrum ihres Denkens steht die menschliche Person als Ganzes. Die Ordo-Liberalen lehnen einen normativen Individualismus ab, demzufolge das Individuum zur Quelle aller Werte gemacht wird. Tatsächlich kritisierten sie „eine rein individualistische Orientierung an der Freiheit als höchster gesellschaftlicher Norm sogar explizit“ (Müller 2007, S. 105): „Nicht die Freiheit ist der personale Höchstwert, welcher den Arbeiten Euckens und der anderen Theoretiker der Freiburger Schule zugrunde liegt, sondern ein (sozial-)ethisches Gerechtigkeitsprinzip in der Gestalt des Kantischen Universalisierungspostulats, das seinerseits gerade nicht individualistisch legitimiert ist." (Ebd., S. 100).

Eucken und die Theoretiker der Freiburger Schule verabsolutieren den Wert der Freiheit gegen das soziale und ethische Prinzip der Gerechtigkeit nicht: „Nicht das Individuum dient hier als letzter normativer Bezugspunkt, sondern eine überindividuell definierte Vorstellung vom Gemeinwohl der Gesellschaft." (Ebd., S. 105). Dieses Menschverständnis, das an den Konzepten des Gemeinwohls und der Gerechtigkeit gemessen wird, stimmt mit dem Personalitätsprinzip der christlichen Sozialethik überein. Von daher ist es nicht übertrieben zu behaupten, dass man das sozialethische Anliegen und die Ordnungstheorie der Freiburger Schule im weiteren Sinne als „eine Spielart der christlichen Sozialethik" (Ebd., S. 106) ${ }^{18}$ verstehen kann.

\footnotetext{
18 Hier gibt es eine Affinität, die auch bei den konkreten wirtschaftspolitischen Folgerungen der Soziallehre feststellbar ist (vgl. Nass und Müller 2013).
} 
Mit dem Ordo-Gedanken verbinden Eucken sowie auch weitere Vertreter des Ordoliberalismus die Suche und die Vorstellung einer Wirtschafts- und Gesellschaftsordnung, die menschenwürdig ist und die Freiheit des Einzelnen gewährleistet. Es geht um eine zu gestaltende und zu realisierende Ordnung, die stets eine Aufgabe für den einzelnen Menschen und die gesellschaftlichen Institutionen darstellt. Oder, wie Böhm sagt, es geht um eine Ordnung, die „durch [die] Freiheit des Planens unter der Herrschaft des Gesetzes" (Böhm 1950 S. XLVIII) gekennzeichnet ist. Böhms Zitat drückt das Wesen von Euckens Ordo-Gedanken aus: Einerseits die Sicherung der menschlichen Freiheit und anderseits die Anforderung, eine gerechte marktwirtschaftliche Ordnung zu schaffen.

Die Verwendung des Ordo-Begriffs als Ordnung bezeichnet bei den Vertretern der Freiburger Schule die Suche einer stabilen konstitutionellen Ordnung für die marktwirtschaftlichen Prozesse. Sie betrachten die Wirtschaftsordnung als eine verfassungsrechtliche Ordnung, die zu verwirklichen ist. Von daher besitzt der Begriff "Ordnung" keine autoritäre Konnotation, sondern er ist mit der Idee einer Konstitutionsökonomik zu verbinden, die als die Durchsetzung von Spielregeln zu verstehen ist. Der Ordo-Begriff spiegelt sich in dem Prinzip der Interdependenz der Ordnungen wider und in der Integration der Wirtschaftsordnung in einen höheren gesellschaftlichen, politischen und rechtlichen Rahmen, der die Wirtschaft lenken und ihr Grenzen setzen soll (Felice 2014).

Der Begriff Ordnung hat einen polysemen Sinn. Er bezeichnet die Notwendigkeit einer Ordnung als Kombination von Regeln (Forte 2010, S. 27), aber auch als ein Ordnungsziel, in dem sich verantwortungsvoll die Entfaltung der menschlichen Person abspielen kann. Der Ordoliberalismus stellt einen alternativen und systematischen Ansatz zum klassischen Liberalismus des Laissez-faire und den Degenerationen des Kapitalismus dar. Weit entfernt vom einem autoritären Sinn des Wortes verweist die Ordnung für die Ordoliberalen auf die Koordination der einzelnen Pläne, auf eine dezentrale Koordinierung der wirtschaftlichen Tätigkeiten innerhalb eines Rahmens von allgemeinen Spielregeln und auf die Ablehnung der Unterwerfung der wirtschaftlichen Aktivitäten unter eine Zentralbehörde (Felice 2009 , S. 10).

\subsection{Die kritische Würdigung der Frage nach dem Naturrecht}

Ein weiteres Thema, das in der Freiburger Denkschrift im Zusammenhang mit dem Ordo-Gedanken steht, ist die Frage nach dem Naturrecht. Die Denkschrift enthält und spiegelt die damalige protestantische sowie ökumenische und juristische Diskussion um die Frage des Naturrechtes wieder (Holthaus 2015, S. 206-221). Dennoch spürt man in ihr auch den Einfluss der römischkatholischen Auffassung. Die meisten Autoren der Denkschritt waren bewusst evangelische Christen, aber an den Treffen des Freiburger Konzils nahmen auch Katholiken teil. Die Behandlung der Frage nach dem Naturrecht scheint in der Denkschrift zweideutig.

Einerseits lehnt die Denkschrift aufgrund der lutherischen Auffassung des Menschenbildes, der Rechtfertigungstheologie und des Sündenverständnisses das Naturrecht ab (Ebd., S. 207 f.). Gegenüber der Auffassung, einige Ordnungen als "gott- und naturgewollt, vernünftig schlechthin und damit auch christlich zu erweisen“, stellt die Denkschrift klar fest: „Aber wir evangelischen Christen müssen es ablehnen, innen irgendeinen absoluten Geltungsanspruch einzuräumen" (In der Stunde Null, S. 56). Trotz dieser Kritik schreibt andererseits die Denkschrift dem Naturrecht ein Wegweiserfunktion für die Suche nach einer gerechten Gemeinschaftsordnung zu (Ebd., S. 59). Darüber hinaus stellt die Denkschrift fest, dass es ein "natürliche[s] Gewissen auch der Nichtchristen“, ein "natürliche[s] Rechtsbewußtsein“ (Ebd., S. 72) gebe.

Wie Stephan Holthaus analytisch gezeigt hat, war die Kritik der Denkschrift am Naturrecht nicht prinzipiell an die katholische Auffassung gerichtet, sondern an den totalitären Staat des Nationalsozialismus und seine Ideologie (Holthaus 2015, S. 209). Die Denkschrift lehnt das ideologische Naturrecht und die natürlichen Ordnungen der nationalistischen Ideologie ab, die versuchten, bestimmte Ordnungen durch den Rekurs auf ein universelles Naturrecht, auf „historische Autoritäten“, „Lebensnotwendigkeiten" oder „Herrschaftsansprüchen der Nationen und Rassen" (In der Stunde Null, S. 59) zu legitimieren. Die Denkschrift räumt dennoch der menschlichen Vernunft eine gemäße Rolle ein: „Die schroffe Ablehnung des Naturrechts richtete sich in erster Linie gegen die Ideologie des Nationalsozialismus, nicht gegen das katholische Naturrechtsverständnis an sich. Denn ein positives Verhältnis zum Naturrecht gab es in einer speziellen Fragestellung der Denkschrift, die überrascht, in Bezug auf den Stellenwert der,Vernunft"“ (Holthaus 2015, S. 210).

Tatsächlich weist die Denkschrift eine rationalistische Auffassung des Naturrechtes und die "scholastische Umdeutung des urchristlichen Mysteriums" (In der Stunde Null, S. 60) zurück. Gleichzeitig behauptet sie jedoch, dass die „Sachverständigkeit“ (Ebd., S. 60), 
das "Salz christlicher Erkenntnis“ (In der Stunde Null, S. 35) und eine „vernünftige Einsicht“ (Ebd., S. 102) notwendig seien. In Bezug auf die Gestaltung der Wirtschaftsordnung sagt die Anlage 4 pointiert, dass sie eine "Sache der menschlichen Vernunft" sei; und dass neben den „biblischen Richtschnuren und Verboten" der Kirche auch "Grundsätze, die sich aus Sachnotwendigkeiten des Wirtschaftens ergeben" (Ebd., S. 128) erforderlich seien.

Obwohl die Denkschrift des Freiburger Kreises prägend von protestantischen Autoren verfasst wurde und die ethische Diskussion der damaligen protestantischen Theologie rezipiert, kann man dennoch Folgendes festhalten: Auf den Inhalt der Denkschrift haben auch katholisch-theologischen Ansätze eingewirkt, wie am Beispiel der Naturrechtskonzeption und des Hinweises auf das Subsidiaritätsprinzip verdeutlicht worden ist. Von daher kann man insgesamt von einer ökumenischen Relevanz des Textes sprechen.

Ein weiterer Diskussionspunkt hinsichtlich der Auffassungen von Eucken betrifft in diesem Zusammenhang die These, dass Eucken kein Anhänger, sondern „ein erklärter Gegner des Naturrechtsdenkens" (Pies 2002, S. 6) sei. Dagegen behauptet Viktor Vanberg, dass, obwohl Euckens Argumentation für die Gestaltung einer menschenwürdigen Wirtschaftsordnung einen Bezug auf naturrechtliche und normative Kriterien enthält, diese Bezüge dennoch nicht seinen Hauptgedanken beeinträchtigen (Vanberg 1997, S. 64). Die normative Ordo-Idee als Ordnung, die zu verwirklichen ist, ist mit der Suche nach den verfassungsrechtlichen und konstitutionellen Grundlagen einer gerechten, funktionsfähigen und menschenwürdigen Wirtschafts- und Gesellschaftsordnung verbunden.

Vanberg betont, dass bei Eucken naturrechtliche und normative Kriterien nicht der Maßstab für die wünschenswerte und menschenwürdige Ordnung sind, weil Euckens Forschungsprogramm auf dem Kriterium des „konsensfähigen konstitutionellen Interesses aller Jurisdiktionsmitglieder" basiert:

„Die relevante Frage ist [...], wie man solche Äußerungen im Kontext des gesamten Forschungsprogramms interpretiert und gewichtet, ob man meint, daß sie konstitutiv für dieses Programm sind, oder ob man darin ein Element sieht, das innerhalb des Forschungsprogramms keine tragende Funktion hat und auf das verzichtet werden kann, ohne daß dadurch das Gesamtargument beeinträchtig würde. Nach meiner Meinung ist letzteres der Fall, [...] daß der Freiburger ordnungsökonomische Ansatz im Sinne eines strikt prozeduralen Liberalismus verstanden werden kann, der in keiner Weise auf naturrechtliche

Argumentationsmuster angewiesen ist." (Ebd., S. 56).

Tatsächlich behauptet Eucken, dass es sich bei den konstituierenden und regulierenden Prinzipien „nicht um rechtsdogmatische und nicht um naturrechtliche Prinzipien" handelt und diese Prinzipien werden nicht aus dem Naturrecht oder aus „übergeordneten rechtsdogmatischen Sätzen“ abgeleitet. Für Eucken gilt: „Auf dem positiven Zweck liegt bei allen diesen Prinzipien der Nachdruck“ (Eucken 2004, S. 290). Und weiterhin schreibt er:

„Die Lösung der Ordnungsfrage ist aber heute nicht mehr in unmittelbarer Erfahrung möglich. Und ebenso wenig in unmittelbaren naturrechtlichen Ableitungen. Eine wissenschaftliche Analyse der Probleme ist erforderlich. In der Wirklichkeit muß die Sachgesetzlichkeit entdeckt werden, deren Kenntnis die Gestaltung der Ordnungen auch in dem von den Kirchen erstrebten Sinne erst ermöglicht.“ (Ebd., S. 347).

Zur Klärung der Bedeutung der normativen und naturrechtlichen Aussage von Euckens Forschungsprogramm hat ebenso wesentlich Nils Goldschmidt beigetragen. Er argumentiert, dass Eucken nicht einer scholastischen oder besser neuscholastischen - naturrechtlichen Auffassung verpflichtet bleibt, nach der aus der Offenbarungswahrheit und den Schöpfungsaussagen durch die natürliche Vernunft die Rechtssätze und die Prinzipien der lex aeterna qua lex naturalis abgeleitet werden. Eucken bestreitet nur die „unmittelbare“ Ableitung von Prinzipien aus dem Naturrechtsdenken: „Diese Vorgehensweise ist für Eucken im Rahmen einer modernen Wissenschaftskonzeption nicht mehr haltbar. [....] Wenn Eucken also unmittelbar naturrechtliche Ableitungen verwirft, berührt dies den metaphysischen Kern seiner Konzeption in keiner Weise." (Goldschmidt 2002, S. 106).

Tatsächlich weisen Euckens Idee gewisse Affinitäten zu der Kritik des katholischen Theologen Joseph Höffner an die neuscholastische Naturrechtslehre auf. Diese letztere Auffassung basierte auf einer rein deduktiven Methode und auf einer rein metaphysischen Begründung, die aus dem göttlichen ewigen Gesetz die Normen des Naturrechts durch wirklichkeitsferne Spekulationen ableiteten. Dabei berücksichtigte die neuscholastische Naturrechtslehre nicht die historischen und soziologischen Erkenntnisse und Analysen der konkreten Verhältnisse (Höffner 1961, S. 207-209).

Höffner setzt sich kritisch mit dieser Auffassung auseinander. Seine Begriffsbestimmung des Naturrechts 
charakterisiert die Idee der Würde des Menschen als Geschöpf und Ebenbild Gottes. Wenngleich Höffner betont, dass das Naturrecht seine Wurzeln in dem göttlichen ewigen Gesetz (lex aeterna) des Schöpfers hat und zum natürlichen Sittengesetz gehört (Höffner 1983, S. 268), berücksichtigt er ebenso diejenigen Einwände und Kritiken, die vor allem gegen das christliche und sozialethische Verständnis des Naturrechts formuliert wurden. Höffner lehnt z.B. die Auffassungen des atheistischen Existenzialismus, des Rechtspositivismus sowie des Rechtssoziologismus ab. Nach diesen Denkströmungen gibt es keine Menschennatur und keine in sich schlechten Handlungen. Diese Tendenzen betonen, dass, obwohl es in den verschiedenen Kulturen allgemeine Rechtsauffassungen gibt, diese „weder unwandelbar noch allgemeingültig, sondern an das ,Gesamtgefüge der jeweiligen Kultur' gebunden“ (Ebd., S. 272) sind.

Ein weiterer umstrittener Kritikpunkt am Naturrecht besagt, dass das Naturrecht die historische Dimension des Menschen und der gesellschaftlichen Zusammenhänge verkennt. Höffner nimmt diesen Einwand ernst, nach dem sich das Naturrecht „Zu einer wirklichkeitsfremden Abstraktion“ verflüchtige (Ebd., S. 275). Im Lichte der Lehre von Thomas von Aquin und des Denkens der spanischen Kolonialethiker antwortet Höffner auf diese Einwände, dass diese Theologen bereits die Unterscheidung zwischen der Unwandelbarkeit der gottgesetzten Normen und der Ordnungen und den historisch wandelbaren ökonomischen und gesellschaftlichen Verhältnissen beachtet haben. Mit Verweis auf Suarez, Thomas und Molina stellt Höffner fest: „Das Naturrecht ist zwar übergeschichtlich gültig, wird aber selbstverständlich nur in der Geschichte wirksam, ist also in dieser Hinsicht keineswegs ,geschichtslos' oder ,übergeschichtlich“ oder ,transzendent', sondern den jeweiligen geschichtlichen Rechtsordnungen immanent. Das Naturrecht ist ein ständig zu verwirklichendes ,Programm“" (Ebd., S. 276).

Zu den Eigenschaften des Naturrechts zählt Höffner unter anderem folgende drei Aspekte: Allgemeingültigkeit, Unwandelbarkeit und Erkennbarkeit. Das Naturrecht ist allgemeingültig in dem Sinne, dass es sich aus der Menschennatur ergibt, die bei allen Menschen gleich ist, und von daher ,jedermann verpflichtet“. Die Erkennbarkeit besteht darin, dass die dem Naturrecht zugrundeliegenden Prinzipien nicht durch formelle Inhalte und Formulierungen, sondern in den konkreten Verhältnissen - vor allem in der Familie - zu erfassen sind, wo der Mensch das Naturrecht „erlebt und erlernt“. Darüber hinaus bezeichnet die Umwandelbarkeit des Naturrechts die Tatsache, dass, während die positiven
Gesetze dem Wandel unterliegen und von den jeweiligen Verhältnissen abhängig sind, dagegen die naturrechtlichen Grundnormen unwandelbar sind, weil sie „auf dem überzeitlich gültigen, metaphysischen Wesenskonstitutiv des Menschen" (Ebd., S. 279) beruhen und damit der Geschichtlichkeit der Menschen nicht widersprechen.

Worin besteht aber heute die bleibende Bedeutung des Naturrechtes und der Menschenwürde? Man kann den Begriff der Menschenwürde im doppelten Sinn verstehen: Einerseits im engeren Sinne als die „Fähigkeit zum freien Handeln und zur eigenverantwortlichen Lebensgestaltung", anderseits im weiteren Sinne als eine anthropologische Einsicht, die zur menschenwürdigen Gestaltung des individuellen und sozialen Lebens verpflichtet. Diese Aspekte bezeichnen einen „minimalen“ normativen Gehalt des Naturrechts und des Begriffes der Menschenwürde (Schockenhoff 2008, S. 240 f.).

Was heute in der wissenschaftlichen Diskussion betont und weiter entfaltet werden sollte, ist nicht die Letztbegründung der Normen bzw. der Menschenrechte, sondern der Inhalt der vorgeschlagenen Normen und der Menschenwürde, d.h. die Forderung nach Freiheit, Privateigentum, Gleichheit und Toleranz. Man kann die intersubjektive Geltung der Forderungen der Menschenrechte und Menschwürde als Anspruch auf allgemeine Anerkennung auffassen, die sich z.B. auf folgenden Grund stützen kann, nämlich, dass soziale Ordnungen, in denen sich solche Forderungen oder moralischen Überzeugungen durchgesetzt haben, zur Verbesserung des Lebens und zur Zufriedenheit der Menschen beigetragen haben (Albert 2013, S. 52). Man kann von daher von normativen Konsequenzen des Ideals der Menschenrechte und des Naturrechtsdenkens sowie „von einem unverzichtbaren humanen Überschuss des Naturrechts“ sprechen (Schockenhoff 2008, S. 240).

Schließlich kann man behaupten, dass sich die inhaltlichen Forderungen des Naturrechts und der Menschenwürde und ihre Umsetzung mit der Frage der Wirtschaftsordnung in Verbindung setzen lassen. Tatsächlich kann man eine Analogie zwischen dem Ziel, die Forderungen des Naturrechtes zu realisieren, und dem Sachziel der Wirtschaft, das in der Schaffung materieller Voraussetzungen zur menschenwürdigen Entfaltung liegt, erstellen (Höffner 1985, S. 356). Analog soll die Menschenwürde als Forderung zur Befriedigung der menschlichen Bedürfnisse durch eine entsprechende Sozialordnung gestaltet werden. Diese normativen Konsequenzen spiegeln sich wieder auf der Ebene der individuellen Tugendethik sowie der 
Rechtspflichten und des politisch-institutionellen Rahmens, die zusammen die Sicherung des Friedens, der Freiheit und der materiellen, geistigen und kulturellen Entfaltungsbedingungen der menschlichen Person gewährleisten sollen.

\section{Fazit und Ausblick}

Für Eucken bezeichnet die Ordo-Idee eine anzustrebende Ordnung, eine Wesensordnung und Naturordnung, die gegen die konkreten ungerechten Ordnungen gerichtet ist. Mit anderen Worten drückt der Ordnungsbegriff die Idee aus, eine „funktionsfähige und menschenwürdige Ordnung der Wirtschaft, der Gesellschaft, des Rechtes und des Staates zu finden." (Eucken 1950, S. 239). Eucken kritisierte auch die neuscholastische Ansicht einer reinen und direkten Ableitung von ethischen Normen aus dem Naturrecht. Der Vater des Ordoliberalismus lehnte die unmittelbare Ableitung von Normen aus dem Naturrecht und der christlichen Offenbarung ab. Jedoch bestritt er die normativen Grundlagen seines eigenen Denkens nicht, sondern er wies ausdrücklich auf sie hin.

Euckens Denken bezeugt darüber hinaus bestimmte Affinitäten zu der christlichen Sozialethik. Zusammen mit anderen ordoliberalen Autoren betonte Eucken die Notwendigkeit einer moralischen Grundlage für die Wirtschaftsordnung. Er betrachtete den Ordo-Gedanken und den christlichen Glauben als normatives Fundament seiner volkswirtschaftlichen Auffassungen. Die Berücksichtigung der Prinzipien der christlichen Sozialethik findet einen vielfältigen Ausdruck in den

\section{Literaturverzeichnis}

Albert, Hans (2013), Zum Problem der Objektivität der Moral, in: Aufklärung und Kritik, Bd. 20/3, S. $46-52$.

Aquin, Thomas von (1956), Summa theologiae, 3 Bde? ediert von Petrus Caramello, Rom-Turin.

Becker, Helmut Paul (1965), Die soziale Frage im Neoliberalismus: Analyse und Kritik, Heidelberg.

Blumenberg-Lampe, Christine (1986), Der Weg in die Soziale Marktwirtschaft: Referate, Protokolle, Gutachten der Arbeitsgemeinschaft Erwin von Beckerath 1943-1947, Stuttgart.

Böhm, Franz (1950), Die Idee des Ordo im Denken Walter Eucken, ORDO - Jahrbuch für die Ordnung von Wirtschaft und Gesellschaft, Vol. 3, S. XVLXIV.
Grundlagen des Ordoliberalismus: In dem rechten Ausgleich zwischen Eigeninteresse und Gemeinwohl; in der Kohäsion und sozialen Integration des Menschen; in einem gesunden Verhältnis zwischen Individuum, Gemeinschaft und Staat; in der Verfolgung von sozialen Zielen und in der Einheit zwischen Wirtschafts- und Gesellschaftsordnung.

Die Vertreter des Ordoliberalismus verteidigten die Würde des Menschen, die freien Initiative und die freie Markwirtschaft, die an den Prinzipien der Personalität, Subsidiarität, Solidarität und Gemeinwohls ausgerichtet werden soll. Sie teilten die Notwendigkeit einer menschenwürdigen Wirtschafts- und Sozialpolitik sowie einer gerechten Wettbewerbsordnung. Gemeinsam forderten sie auch die Verankerung und Einbettung der marktwirtschaftlichen Ordnung in einen ethischen, rechtlichen und institutionellen Rahmen.

Eucken führte die Notwendigkeit für die katholische und protestantische Kirche an, sich über wirtschaftlichen Fragen zu äußern. Dennoch betonte er gleichzeitig die Grenzen der kirchlichen Kompetenz bei solchen Fragen. Die Vertreter des Ordoliberalismus teilten mit der christlichen Soziallehre bestimmte Annahmen bezüglich der Zuständigkeit der Kirche bei wirtschaftlichen, gesellschaftlichen und politischen Fragen. Beide wiesen darauf hin, dass die Kirche keine praktische und technische Lösung zu ökonomischen und sozialen Problemen anzubieten hat, jedoch normative Orientierungen geben kann. Beide Ansätze teilen die Idee, dass die Kirche den Laien die Aufgabe zur konkreten Gestaltung der Wirtschafts- und politische Ordnung überlassen soll.
Bücher, Karl (1925), Die Entstehung der Volkswirtschaft, II. Sammlung, Tübingen.

Dürr, Ernst-Wolfram (1954), Wesen und Ziele des Ordoliberalismus, Winterthur.

Eucken, Walter (1932), Walter Eucken: Religion Wirtschaft - Staat: Zur Problematik des Gegenwartsmenschen, in: Die Tatwelt, Bd. 8, S. 82-89.

Eucken, Walter (1950), Die Grundlagen der Nationalökonomie, Berlin.

Eucken, Walter (2004), Grundsätze der Wirtschaftspolitik, Tübingen.

Felice, Flavio (2009), Luigi Sturzo e l'economia sociale di mercato, in: Studi economici e sociali, Bd. 44/4, S. 9-26. 
Felice, Flavio (2014), Persona, istituzioni e mercato. La persona nel contesto del liberalismo delle regole, Soveria Mannelli.

Forte, Francesco (2010), Introduzione zu: Forte, Francesco und Felice, Flavio (Hg.), II liberalismo delle regole. Genesi ed eredità dell'economia sociale di mercato, Soveria Mannelli S. 25-43.

Franco, Giuseppe (2015), Da Salamanca a Friburgo. Joseph Höffner e l'Economia Sociale di Mercato, (Corona Lateranensis, Bd. 66), Città del Vaticano.

Franco, Giuseppe (2016), Etica senza economia? ॥ contributo di Wilhelm Röpke all'etica economica e al pensiero sociale cristiano, Soveria Mannelli, S. 293-318.

Gässler, Gregor Fidelis (1950/1994), Der Ordo-Gedanke unter besonderer Berücksichtigung von Augustinus und Thomas von Aquino, Sankt Augustin.

Goldschmidt, Nils (2002), Entstehung und Vermächtnis ordoliberalen Denkens. Walter Eucken und die Notwendigkeit einer kulturellen Ökonomik, Münster.

Goldschmidt, Nils (Hg.) (2005a), Wirtschaft, Politik und Freiheit. Freiburger Wirtschaftswissenschaftler und der Widerstand, Tübingen.

Goldschmidt, Nils (2005b), Die Rolle Walter Euckens im Widerstand: Freiheit, Ordnung un Wahrhaftigkeit als Handlungsmaximen, in: Goldschmidt, Nils (Hg.), Wirtschaft, Politik und Freiheit. Freiburger Wirtschaftswissenschaftler und der Widerstand, Tübingen, S. 289-314.

Goldschmidt, Nils (2008), Zur Einführung: Wirtschaftsund Sozialordnung, in: Goldschmidt, Nils und Wohlgemuth Michael (Hg.), Grundtexte zur Freiburger Tradition der Ordnungsökonomik, Tübingen, $\mathrm{S}$. 124-129.

Goldschmidt, Nils und Ursula Nothelle-Wildfeuer (Hg.) (2010), Freiburger Schule und Christliche Soziallehre: Joseph Kardinal Höffner und die Ordnung von Wirtschaft und Gesellschaft, Tübingen.

Goldschmidt, Nils und Michael Wohlgemuth (Hg.) (2008), Grundtexte zur Freiburger Tradition der Ordnungsökonomik, Tübingen.

Hensel, Karl Paul (1949), Ordnungspolitische Betrachtungen zur katholischen Soziallehre (im Sinne der päpstlichen Enzykliken Rerum novarum und Quadragesimo anno), ORDO, Vol. 2, S. 229-269.

Hippo, Augustinus von (1955), De civitate Dei, editiert von Bernhard Dombart und Alfons Kalb, Turnholt 1955 (Corpus Christianorum, series Latina, 47-48).
Holthaus, Stephan (2015), Zwischen Gewissen und Gewinn: Die Wirtschafts- und Sozialordnung der „Freiburger Denkschrift“ und die Anfänge der Sozialen Marktwirtschaft, Berlin.

Höffner, Joseph (1941), Wirtschaftsethik und Monopole im fünfzehnten und sechzehnten Jahrhundert, in: Höffner, Joseph (Hg.) (2014): Wirtschaftsordnung und Wirtschaftsethik. (Ausgewählte Schriften, Bd. 3), Paderborn, S. 33-188.

Höffner, Joseph (1961), Johannes Messner und die Renaissance des Naturrechts, in: Höffner, Joseph (Hg.) (2015), Perspektiven sozialer Gerechtigkeit (Ausgewählte Schriften, Bd. 1), Paderborn, S. 200-213.

Höffner, Joseph (1983), Christliche Gesellschaftslehre, in: Höffner, Joseph (Hg.) (2015), Perspektiven sozialer Gerechtigkeit (Ausgewählte Schriften, Bd. 1), Paderborn, S. 239-481.

Höffner, Joseph (1985), Wirtschaftsordnung und Wirtschaftsethik, in: Höffner, Joseph (2014), Wirtschaftsordnung und Wirtschaftsethik (Ausgewählte Schriften, Bd. 3), Paderborn, S. 337-377.

In der Stunde Null: Die Denkschrift des Freiburger „Bonhoeffer-Kreises“: Politische Gemeinschaftsordnung. Ein Versuch zur Selbstbesinnung des christlichen Gewissens in den politischen Nöten unserer Zeit, Tübingen 1979.

Krings, Hermann (1941/1982), ORDO. Philosophischhistorische Grundlegung einer abendländischen Idee, Hamburg.

Lenel, Hans Otto (1991), Walter Euckens Briefe an Alexander Rüstow, ORDO, Vol. 42, S. 11-14.

Miksch, Leonhard (1937), Wettbewerb als Aufgabe, Stuttgart und Berlin.

Müller-Armack, Alfred (1959), Religion und Wirtschaft. Geistesgeschichtliche Hintergründe unserer europäischen Lebensform, Stuttgart.

Müller, Christian (2007), Neoliberalismus und Freiheit. Zum sozialethischen Anliegen der Ordo-Schule, ORDO, Vol. 58, S. 99-108.

Nass, Elmar und Christian Müller (2013), Normative Grundlagen des Ordoliberalismus, in: Pribyl, Herbert (Hg.), Die Weltwirtschaftskrise. Lösungsansätze aus christlich-ethischer Sicht, Heiligenkreuz, S. 157-183.

Nawroth, Egon Edgar (1961), Die Sozial- und Wirtschaftsphilosophie des Neoliberalismus. Heidelberg-Löwen.

Nell-Breuning, Oswald von (1954), Gemeinsames und Trennendes in den Hauptrichtungen der Wirtschaftswissenschaft und Wirtschaftspolitik, in: Kosiol, Erich und Paulsen, Andreas (Hg.), 
Grundsatzfragen der Wirtschaftsordnung, Berlin, S. $215-231$

Oswalt, Walter (2005), Liberale Opposition gegen den NS-Staat: Zur Entwicklung von Walter Euckens Sozialtheorie, in: Goldschmidt, Nils (Hg.), Wirtschaft, Politik und Freiheit. Freiburger Wirtschaftswissenschaftler und der Widerstand, Tübingen, S. 315-353.

Pies, Ingo (2001), Eucken und Hayek im Vergleich. Zur Aktualisierung der ordnungspolitischen Konzeption, Tübingen.

Ritter, Joachim und Karlfried Gründer (Hg.) (1984), Historisches Wörterbuch der Philosophie, Bd. VI, Basel.

Schockenhoff, Eberhard (2008), Stärken und innere Grenzen. Wie leistungsfähig sind naturrechtliche Ansätze in der Ethik?, Herderkorrespondenz, Bd. 62, S. 236-241.

Schwabe, Klaus und Rolf Reichardt (1984), Gerhard Ritter. Ein politischer Historiker in seinen Briefen, Boppard am Rhein.

Secci, Alessandra (1998), Una resistenza a metà. L'esperienza di opposizione antinazista dei Circoli friburghesi, in: Italia contemporanea, Bd. 211, S. 347-370.

Sombart, Werner (1911), Die Juden und das Wirtschaftsleben, Leipzig.

Starbatty, Joachim (1997), Soziale Marktwirtschaft als Forschungsgegenstand. Ein Literaturbericht, in: Ludwig-Erhard-Stiftung e.V. (Hg.), Soziale Marktwirtschaft als historische Weichenstellung. Bewertungen und Ausblicke. Eine Festschrift zum hundertsten Geburtstag von Ludwig Erhard, Düsseldorf, S. 63-99.

Vanberg, Viktor J. (1997), Die normativen Grundlagen von Ordnungspolitik, in: Vanberg, Viktor J. (2008), Wettbewerb und Regelordnung, Tübingen, S. 49-67.

Veit, Otto (1953), Ordo und Ordnung. Versuch einer Synthese, ORDO, Vol. 5, S. 3-47.

Voigt, Stefan (1996), Die konstitutionelle Ökonomik als Herausforderung für die Theorie der Wirtschaftspolitik. Zugleich eine Skizze zur Weiterentwicklung einer ökonomischen Theorie der Verfassung, in: Pies, Ingo und Leschke, Martin (Hg.), James Buchanans konstitutionelle Ökonomik, Tübingen, S. 157-192. 